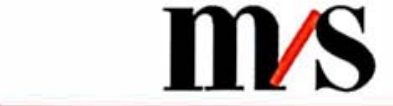

médecine/sciences $1991 ; 7: 440-2$

\title{
TRAITEMENTS MÉDICAUX DES CANCERS : LES NOUVELLES VOIES
}

\section{Dominique Belpomme}

\section{ADRESSE}

D. Belpomme : praticien hospitalier, directeur du centre de recherche de l'ARTAC (association pour la recherche thérapeutique anticancéreuse). Unité de chimiothérapie, hôpital Bichat, 46,

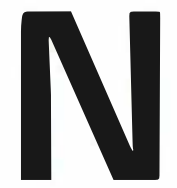

ée après la dernière guerre, et d'abord utilisée essentiellement dans les leucémies et les lymphomes, la chimiothérapie a vu, depuis ces dernières années, sa pharmacopée se diversifier et ses indications s'élargir. C'est vers les années 1975 que nous avons appliqué pour la première fois les concepts de l'hématologie tumorale au traitement des cancers solides [1,2]. Quinze ans plus tard, il est possible de mesurer le chemin parcouru. Force est de reconnaître que si d'importants progrès ont été obtenus dans les lymphomes, les leucémies et certains cancers solides (embryonnaires ?) de l'enfant et de l'adulte jeune, la plupart des cancers communs de l'adulte, de très loin les plus fréquents, sont demeurés jusqu'à maintenant dans l'ensemble, peu perméables à ces progrès. C'est dire l'intérêt de ce numéro de médecine/sciences conçu au décours du troisième congrès de l'ARTAC et consacré aux traitements médicaux des cancers et à leurs nouvelles perspectives.

La chimiothérapie, telle que nous l'entendons classiquement, relève d'un phénomène de cytotoxicité, résultant de l'induction de lésions irréversibles au niveau de l'ADN tumoral, par mécanisme direct (incorporation, adduction) ou indirect (inhibition enzymatique, altération protéique), bien que pour certains médicaments d'autres cibles cellulaires puissent être concernées [3]. Le résultat en est l'inhibition de synthèse de l'ADN et/ou l'altération des processus de division, lesquelles conduisent le plus souvent à la mort cellulaire. Dans l'article : "Diversité des mécanismes de résistance aux chimiothérapies anticancéreuses " (p. 465), j'analyse les mécanismes d'échappement cellulaire aux phénomènes de cytotoxicité classique. Si les caractéristiques cinétiques de croissance tumorale et le point d'impact des différents agents cytotoxiques sur le cycle cellulaire ont longtemps conditionné la pratique des chimiothérapies, une telle approche n'a le plus souvent pas permis de lever l'obstacle des résistances observées cliniquement. Le concept purement cinétique de la résistance ne peut donc en résumer la complexité. L'existence d'un stroma, et son altération possible par la radiothérapie ou même la chimiothérapie, représentent une contrainte spécifique aux cancers solides. L'altération précoce du stroma explique sans doute leur moindre perméabilité aux agents cytotoxiques et peut-être leur plus grande chimiorésistance. Cependant, la très grande diversité des mécanismes de résistance intrinsèque témoigne aussi de l'extraordinaire plasticité des cellules tumorales à résister à ces agents. Interviennent non pas tant leur inactivation métabolique au niveau des cellules tumorales et/ou de l'organisme que surtout l'activation des processus de détoxification et de réparation cellulaire. Or, cette très grande diversité de mécanismes contraste avec la très grande fréquence des résistances secondaires à leur combinaison (pléiotropie). A la vue classique, selon laquelle ces phénomènes résultent d'une sélection d'événements mutationnels aléatoires, nous proposons de substituer, ou plutôt d'ajouter, un processus d'induction directe des mécanismes de résistance par les agents cytotoxiques eux-mêmes [5]. En témoigne l'induction de mutation et d'amplification géni- 
ques. En témoigne aussi la capacité de ces agents à moduler l'expression génique (et donc phénotypique) des cellules tumorales par des mécanismes épigénétiques ou extragénétiques $[5,6]$. Une telle hypothèse inductive devrait donc conduire à un nouveau typc de recherches visant à contourner les phénomènes de résistance.

C'est une voie originale, proche de la réflexion précédente, que proposent L. Degos et al. (p. 460). Dans la cellule cancéreuse, les programmes génétiques de division et de différenciation ne sont qu'incomplètement découplés. La possibilité d'un certain degré de différenciation persiste. Rétablir un état de différenciation suffisant pour conduire la cellule à sa mort naturelle par un phénomène d'apoptose, tcl est l'objectif thérapeutique quc nous propose déjà dcpuis plusieurs années L. Sachs. Les leucémics se prêtent particulièrement bien à ce typc d'approche. Dans un travail précédent, L. Degos avait montré que la cytosinc arabinoside utilisée à faible dose est capable d'induire la régression complètc de certaines leucémies aiguës myéloïdes. La possibilité, même à ces doses, de phénomènes de cytotoxicité "classique " ne peut cependant être exclue. Reprenant les travaux de M.E. Huang, l'équipe de Saint-Louis apporte aujourd'hui la preuve que l'acide rétinoïque tout-trans permet d'obtenir la régression complète dc leucémics aiguës promyélocytaires, par un mécanisme autre que celui de la cytotoxicité classique. Si le taux de réponses est très élevé, la médiane de durée de celles-ci n'est cependant que de huit mois, ce qui justifie les essais en cours d'association aux chimiothérapies classiques.

Il faut remarquer, cependant, que certains agents cytotoxiques sont cuxmêmes susceptibles d'induire des phénomènes de différenciation [7]. Des études comparatives ultérieures sont donc indispensables pour établir la supériorité de l'association des chimiothérapies classiques à un "différenciateur" comme l'acide rétinoïque. L'action spécifique de ce dernier dans la leucémie aiguë à promyélocytes est certainement liée à l'anomalie moléculaire associée au remanie$\mathrm{m} / \mathrm{s} n^{\circ} 5$, vol. 7 , mai 91 ment chromosomique, marqueur de cette maladie, la formation d'un gènc hybride comportant le domaine de liaison à l'ADN et de fixation du ligand du récepteur $\alpha$ de l'acide rétinoïque. C'est cette particularité qui distingue l'effet de l'acide rétinoïque dans la leucémie promyélocytaire de son influence anciennement étudiée sur des cancers épidermoïdes ou des métaplasies malpighiennes bronchiques [8], voire de l'utilisation d'autres ligands de récepteurs de même type que ceux de l'acide rétinoïque, par exemple le tamoxifène utilisé dans les cancers du sein.

Les possibilités de moduler l'expression des gènes de croissance (oncogènes) au niveau transcriptionnel ou post-transcriptionnel par les interférons [9] constituent une autrc voie thérapeutique, proche de la précédente. L'interféron $\alpha$ a été utilisé dès 1983 par G. Talpaz dans les lcucémics myéloïdes chroniques. F. Guilhot ( $p$. 453) nous dresse le bilan des résultats obtenus depuis ces dernières années. Les taux de réponse sont élevés, aboutissant, dans 5 à $20 \%$ des cas, à des rémissions complètes, matérialisées par la disparition du chromosome Philadelphie. Bicn que les résultats à plus long terme, associant l'interféron $\alpha$ aux chimiothérapics classiques par l'hydroxyurée ou le busulfan, ou à la cytosinc arabinoside, nécessitent d'être évalués dans le cadre d'essais comparatifs, on nc peut qu'insister ici sur l'importance des résultats obtenus, le traitement par l'interféron étant pratiquement le seul, hormis la greffe dc moelle osseuse, à permettre une disparition du clone marqué par la présence du chromosome Philadelphie. En fait, la récente mise à la disposition des cliniciens de nouvelles molécules issues du génie génétique a profondément modifié nos concepts thérapeutiques. L'interféron recombinant $\alpha$ est un exemple de ces progrès, tout comme les différents facteurs de croissance hématopoïétiques (érythropoïétine, G-CSF, M-CSF, GM-CSF, interleukines 1, 3, 4 et 6), qu'étudient J.D. Tigaud, Y. Bastion et B. Coiffier dans leur article (p. 444). Certains facteurs, tels l'érythropoïétine et le G-CSF, stimu- lent la production d'un type cellulaire particulier alors que d'autres, tcls l'IL-3 et le GM-CSF, en stimulent plusieurs. L'utilisation de ces factcurs (érythropoiétinc, G-CSF, GM-CSF), encore du domaine de l'expérimentation clinique, suggère qu'ils ont un effet favorable, dépcndant de la dose, sur l'anémie, la neutropénie et les infections de malades attcints de SIDA (traités par la zidovudine), de syndrome myélodysplasique ou de leucémie aiguë myéloïde. La présence de récepteurs du GM-CSF et/ou du G-CSF sur les cellules leucémiques impose cependant une très grande prudence, compte tenu du risque de stimulation de la prolifération tumorale.

D'autrc part, l'intérêt potenticl de ces facteurs réside dans leur utilisation en association aux chimiothérapics cytotoxiques utilisées dans le traitement des tumeurs solides. L'amélioration de la qualité de vie des malades scmble actucllement établie (diminution des périodes de ncutropénic postchimiothérapcutique, du nombre d'épisodes fébriles, etc.), mais plusieurs problèmes demeurent [10].

La présence des réceptcurs de ces factcurs sur les cellules de ccrtains cancers solides impose la même prudence que pour les leucémics. Les facteurs GM-CSF et G-CSF n'ayant pas d'effet anti-thrombopéniant, lcur association à d'autres molécules, tclle l'IL-3, devra être cnvisagée. Enfin, et surtout, la valeur réelle des intensifications chimiothérapiques en cancérologie reste à préciser. Bicn que des résultats encourageants aicnt pu être obtenus, ricn n'indique encore aujourd'hui que l'augmentation des taux de réponse observés se traduiront par un gain en survic.

Le transfert des technologics et des concepts de la biologie à la clinique, l'un des objectifs majcurs de l'ARTAC, était aussi l'un des thèmes de son troisième congrès. Encore doit-on ici tenir compte des problèmes éthiques posés. C'est ce qu'aborde C. Jasmin dans son article consacré au problème du consentement éclairé, ou plutôt à celui de l'éclairage de ce consentement (p. 473). Avec le cancer et la réalisation des essais cliniques, c'cst, en 
réalité, entre le malade et son médecin, deux dénégations qui s'interpellent, dans un redoutable "jeu de la vérité ". Le médecin, conscient de ses limites, est tenté de voir au-delà de l'intérêt de son malade et de lui proposer un essai thérapeutique destiné à enrichir ses connaissances. A l'inverse, le malade, refusant le plus souvent d'accepter l'inéluctable, qu'il soit ou non capable d'en juger, n'a souvent d'autre perception que la sienne, celle d'un être essentiel, toujours prioritaire dans le combat qu'il mène. Le médecin peut-il rompre la situation précédente, en révélant la vérité qu'il pressent, pour éclairer sa démarche et justifier l'essai thérapeutique qu'il propose ? Tels sont les très difficiles problèmes mis en exergue par la récente loi "Huriet " de protection des personnes. Au colloque singulier et secret, malade-médecin, la rencontre d'une âme et d'une conscience, comme cela nous a été enseigné depuis Hippocrate, s'est peu à peu substituée la loi, autrement dit la défense légale du malade mais aussi la notion d'attitude médicale contrôlable par le droit. Cheminer entre ce qu'exige la loi - la signature d'un consentement éclairé - et ce que veut entendre le malade, et donc ce que peut dire le médecin par respect pour lui, tel est l'enjeu et son étroitesse. Une nouvelle pédagogie médicale, mais aussi sans doute une certaine tolérance dans l'interprétation des textes, sont ici indispensables si l'on veut que la loi ne freine pas la recherche clinique. C'est en effet à celle-ci que l'on doit la mise en évidence des progrès thérapeutiques réalisés. Alors que les frontières de la chimiothérapie, de l'hormonothérapie et de l'immunothérapie semblaient être bien individualisées, celles-ci tendent aujourd'hui à se confondre. Les résultats précédents conduisent à individualiser au moins deux mécanismes essentiels de cytotoxicité : l'un, classique, résultant de l'induction directe ou indirecte de lésions au niveau de l'ADN tumoral, et l'autre, plus physiologique, résultant essentiellement du contrôle négatif des gènes de croissance, et conduisant au vieillissement puis à la mort cellulaire par apoptose, dans le cadre de processus de différenciation et/ou de rétroversion phénotypique. C'est par ce deuxième mécanisme qu'agiraient certains agents cytotoxiques classiques utilisés à faible dose, mais aussi et surtout, des hormones ou agents " hormonaux ", tels le tamoxifène, les acides rétinoïques et l'interféron $\alpha$

\section{RÉFÉRENCES}

1. Pouillart P, Schwarzenberg L, Palangie T, Belpomme D, Mathé G. Associations chimiothérapiques dans le traitement des cancers du sein polymétastasés. Nouv Press Med 1976 ; $5: 21-45$.

2. Belpomme D. Cancérologie générale. Paris : J.B. Baillière, 1979 : 353.

3. Liley DT, Wiggins PM, Bagulley BC. Localisation of a non intercalative DNA binding antitumour drug in mitochondria : relationship to multidrug resistance. Eur $J$ Cancer Clin Oncol 1989; 25 : 1287-93.

4. Belpomme D, Heritier F, Ressayre C. Goldie-Coldman hypothesis revisited : an induction-selection theory of chemoresistance. Proc Am Soc Clin Oncol 1989; $8: 81$.

5. Futscher BW, Erickson LC. Changes in $\mathrm{C}$-myc and C-fos expression in a human tumor cell line following exposure to bifunctional alkylating agents. Cancer Res 1990 ; $50: 62-6$.

6. Nahagawara A, Kadomatsu K, Sato SI, et al. Inverse correlation between expression of multidrug resistance gene and $\mathrm{N}$-myc oncogene in human neuroblastomas. Cancer Res 1990 ; 50 : 3043-7.

7. Bloch A. Induction of tumor cell differentiation as a common mechanism of action of DNA specific antitumor agents. In : Tapiero H, Robert J, Lampidis TJ, eds. Anticancer drugs, vol. 191. Paris: Inserm/John Libbey Eurotext, 1989 : 145-51.

8. Lippman SM, Kessler JF, Meyskens FL. Retinoids as preventive and therapeutic anticancer agents. Cancer Treat Rep 1987 ; 71 : 391-405 (part I) and 493-515 (part II).

9. Clemens M. Interferons and oncogenes. Nature 1985 ; 313 : 521-2.

10. Griffin JD. Hemopoietins in oncology : factoring out myelosuppression. J Clin Oncol $1989 ; 7: 151-5$.

\section{TIRÉS A PART}

D. Belpomme. 